\title{
Coordinated peak expression of MMP-26 and TIMP-4 in preinvasive human prostate tumor
}

Seakwoo Lee ${ }^{1, *}$, Kevin K Desai ${ }^{1, *}$, Kenneth A Iczkowski ${ }^{2,3, *}$, Robert G Newcomer ${ }^{1}$, Kevin J Wu ${ }^{4}$, Yun-Ge Zhao ${ }^{1}$, Winston W Tan ${ }^{5}$, Mark D Roycik ${ }^{1}$, Qing-Xiang Amy Sang ${ }^{1}$

${ }^{I}$ Department of Chemistry and Biochemistry and Institute of Molecular Biophysics, Florida State University, Tallahassee, FL 32306, USA; ${ }^{2}$ Department of Pathology and Laboratory Medicine, Veterans Affairs Medical Center, Gainesville, FL 32608, USA; ${ }^{3}$ Department of Pathology, Immunology, and Laboratory of Medicine, University of Florida College of Medicine, Gainesville, FL 32610, USA; ${ }^{4}$ Department of Pathology, Medicine, Mayo Clinic, Jacksonville, FL 32224, USA; ${ }^{5}$ Department of Internal Medicine, Hematology/Oncology, Mayo Clinic, Jacksonville, FL 32224, USA

The identification of novel biomarkers for early prostate cancer diagnosis is highly important because early detection and treatment are critical for the medical management of patients. Disruption in the continuity of both the basal cell layer and basement membrane is essential for the progression of high-grade prostatic intraepithelial neoplasia (HGPIN) to invasive adenocarcinoma in human prostate. The molecules involved in the conversion to an invasive phenotype are the subject of intense scrutiny. We have previously reported that matrix metalloproteinase-26 (MMP-26) promotes the invasion of human prostate cancer cells via the cleavage of basement membrane proteins and by activating the zymogen form of MMP-9. Furthermore, we have found that tissue inhibitor of metalloproteinases-4 (TIMP-4) is the most potent endogenous inhibitor of MMP-26. Here we demonstrate higher $(p<0.0001)$ MMP-26 and TIMP-4 expression in HGPIN and cancer, compared to non-neoplastic acini. Their expression levels are highest in HGPIN, but decline in invasive cancer $(p<0.001$ for each) in the same tissues. Immunohistochemical staining of serial prostate cancer tissue sections suggests colocalization of MMP-26 and TIMP-4. The present study indicates that MMP-26 and TIMP-4 may play an integral role during the conversion of HGPIN to invasive cancer and may also serve as markers for early prostate cancer diagnosis.

Cell Research (2006) 16:750-758. doi: 10.1038/sj.cr.7310089; published online 29 Aug 2006

Keywords: matrix metalloproteinase-26, tissue inhibitor of metalloproteinases-4, high-grade prostatic intraepithelial neoplasia, prostate cancer biomarkers, early diagnosis, immunohistochemistry, co-immunoprecipitation

\section{Introduction}

The defining phenotype of malignancy is the cells' ability to invade the surrounding stroma and metastasize. This

\footnotetext{
*These three authors contributed equally to this work. Correspondence: Qing-Xiang Amy Sang

Tel: +1-850-644-8683; Fax: +1-850-644-8281;

E-mail: sang@chem.fsu.edu

Abbreviations: matrix metalloproteinase (MMP); tissue inhibitor of metalloproteinases-4 (TIMP-4); high-grade prostatic intraepithelial neoplasia (HGPIN); co-immunoprecipitation (co-IP); extracellular matrix (ECM); ductal carcinoma in situ (DCIS); infiltrating ductal carcinoma (IDC); atypical intraductal hyperplasia (AIDH); Tris-buffered saline (TBS)

Received 8 Mar 2006; revised 9 Jul 2006; accepted 13 Jul 2006; published online 29 Aug 2006
}

requires the degradation of several components of the extracellular matrix (ECM). Matrix metalloproteinases (MMPs) are a family of zinc-catalyzed proteolytic enzymes known to digest the basement membrane, ECM components, and cell surface proteins [1]. MMPs have been linked with cancer cell invasion, growth, angiogenesis, inflammation, and metastasis [1-5]. In addition, MMPs have been shown to play a role in the release of growth factors that can in turn contribute to the invasiveness and growth of tumors [6].

Endometase/matrilysin-2 (MMP-26) is a member of the MMP family recently cloned by our group and others [710]. MMP-26 is one of the two smallest members of this family, exhibiting minimal domain structure consisting of a catalytic domain and a prodomain, which maintains the enzyme in a latent form prior to its activation. Once active, 
MMP-26 has been shown to cleave multiple components of the ECM, including fibronectin, type IV collagen, vitronectin, gelatins, and fibrinogen, as well as non-ECM proteins such as insulin-like growth factor-binding protein1 and $\alpha-1$ protease inhibitor [7-11]. MMP-26 is also able to activate progelatinase B (pro-MMP-9), an enzyme that plays a critical role in ECM remodeling [12].

MMP-26 mRNA is primarily expressed in epithelial cancers, such as lung, breast, endometrial, and prostate carcinomas [7-10]. Our previous studies have shown that MMP-26 expression in human prostate carcinoma is significantly higher than that in prostatitis, benign prostate hyperplasia (BPH), and normal prostate tissue [12]. In addition, our group has shown that the expression of MMP-26 in human breast tissue is significantly higher during preinvasive ductal carcinoma in situ (DCIS) when compared to infiltrating ductal carcinoma (IDC), atypical intraductal hyperplasia (AIDH), and normal breast epithelia adjacent to DCIS and IDC [13]. These results suggest that MMP-26 plays an important role in the early stage prior to the development of invasive breast and prostate cancers.

The activity of MMPs is regulated locally by specific tissue inhibitors of metalloproteinases (TIMPs). Four members of the human TIMP family have been identified as follows: TIMP-1, TIMP-2, TIMP-3, and TIMP-4 [14-17]. The cleavage of synthetic peptides in vitro by MMP-26 is inhibited by TIMP-1, TIMP-2, and TIMP-4, with TIMP-4 displaying the greatest inhibitory potency $[7,13]$. TIMP-4 is a tight-binding and slow-binding inhibitor of MMP-26, with an apparent $K_{\mathrm{i}}$ value of $0.62 \mathrm{nM}$ [13]. TIMP-4 mRNA has been detected in a variety of normal tissues, including those of the heart, kidney, pancreas, colon, testis, endometrium, and placenta [17-19]. Under normal conditions, MMPs and TIMPs are expressed at low levels in most adult tissues, but may become upregulated in pathophysiologic conditions such as wound healing and tumor progression. Mimicking the expression pattern of MMP-26, TIMP-4 expression in human breast DCIS is significantly higher than that detected in IDC, AIDH, and in normal breast epithelium adjacent to DCIS and IDC [13]. The therapeutic potential of TIMP-4 in the treatment of malignant progression has been examined, and the transfection of TIMP-4 cDNA into human MDA-MB-435 breast cancer cells inhibited tumor cell invasion across Matrigel, a barrier of reconstituted basement membrane components [20]. Incubation of these same cells with recombinant TIMP-4 protein displayed a similar inhibitory effect [21].

Much of the cumulative data relating to TIMPs and MMPs in prostate cancer indicate that TIMP expression decreases in cancer, while the ratio of MMPs to TIMPs increases. TIMP-1, -2 , and -3 mRNA molecules and proteins were detected in human prostate cancer specimens
[22, 23], and TIMP-1 has been shown in various studies to be downregulated in prostate cancer as compared to normal prostate $[22,24]$. A study of TIMP-1, -2 , and -3 mRNA showed significant reduction of TIMP-2 and -3 in prostate cancer, as well as significant increases in the ratios of MMP-9 to TIMP-1, -2 , and -3 , and in the ratio of MMP-14 to TIMP-3 [23].

Here, we explore the expression of MMP-26 and TIMP4 in human prostate tissue. We show that the expression of MMP-26 and its most potent endogenous inhibitor, TIMP-4, are highest in preinvasive high-grade prostatic intraepithelial neoplasia (HGPIN), and that levels of the two proteins decline significantly in adjacent areas of cancer in the same tissues. Moreover, immunohistochemical staining of serially sectioned prostate cancer tissues suggests colocalization of MMP-26 and TIMP-4. These results are analogous to our previous findings in human breast cancer showing that MMP-26 is closely coordinated with TIMP-4, with both proteins showing maximal expression in preinvasive DCIS. The combined data from these investigations indicate that MMP-26 and TIMP-4 may be involved in early carcinoma development and the transformation to an invasive phenotype.

\section{Materials and Methods}

\section{Specificity of antibodies}

The two MMP-26 antibodies utilized for this study are directed against either the human MMP-26 prodomain sequence of $\mathrm{Thr}^{50}$ Gln-Glu-Thr-Gln-Thr-Gln-Leu-Leu-Gln-Gln-Phe-His-Arg-AsnGly-Thr-Asp ${ }^{67}-\mathrm{NH}_{2}$ or the C-terminal sequence of $\mathrm{Gln}^{246}$-Arg-IleGln-His-Leu-Tyr-Gly-Glu-Lys-Cys-Ser-Ser-Asp ${ }^{259}-\mathrm{NH}_{2}$. No peptide with $>45 \%$ level of identity to these selected sequences was found as determined using the BLAST search method at the National Center for Biotechnology Information website (http://ncbi.nih.gov/ BLAST/). These rabbit polyclonal and mono-specific antibodies were tested and verified to be highly specific for MMP-26 [12]. A goat polyclonal antibody against the $\mathrm{C}$-terminal sequence region of MMP26 (E-14) was obtained from Santa Cruz Biotechnology, and was utilized for the detection of MMP-26 following immunoprecipitation. A mouse monoclonal antibody against MMP-9 (Ab-1) was obtained from Calbiochem (San Diego, CA, USA). The TIMP-4 antibody was directed against the sequence $\mathrm{Ser}^{61}$-Ala-Asp-Pro-Ala-Asp-ThrGlu-Lys-Met-Leu-Arg-Tyr-Glu ${ }^{74}-\mathrm{NH}_{2}$. This sequence also shares less than $45 \%$ homology with any other known peptide. This rabbit polyclonal and mono-specific antibody was purified, characterized, and its specificity validated as described previously [25].

\section{Western blot}

Proteins were extracted from human malignant prostate tissue using T-PER (Pierce, Rockford, IL, USA). Tissue samples were homogenized with T-PER ( $2 \mathrm{ml}$ per $0.1 \mathrm{~g}$ of tissue), and after centrifugation $(10000 \times g, 5 \mathrm{~min})$, the supernatant was collected. Tissue extracts were subjected to SDS-PAGE, and then blotted onto nitrocellulose blotting membranes (Pall Life Sciences, Pensacola, FL, USA). Membranes were blocked in Tris-buffered saline (TBS) 
solution containing $0.25 \%$ Tween 20 and $5 \%$ bovine serum albumin prior to incubation with primary antibodies. For the detection of MMP-26 directly from tissue extracts, rabbit anti-MMP-26 antibodies were used. For the detection of MMP-26 following immunoprecipitation, a goat anti-MMP-26 antibody (E-14) was used. Membranes were incubated with alkaline phosphatase-conjugated secondary antibodies for $30 \mathrm{~min}$ at room temperature, and positive bands were visualized using NBT/BCIP substrates (Fisher Biotech, Fairlawn, NJ, USA).

\section{Co-immunoprecipitation (co-IP)}

To examine the interaction between MMP-26 and TIMP-4, prior to treatment of prostate cancer tissue extract with anti-TIMP-4 antibody, preimmune IgG was treated with prostate cancer tissue extract to minimize nonspecific interactions as follows. One milliliter of tissue extract was incubated with $1 \mu \mathrm{g}$ of rabbit preimmune IgG and $20 \mu \mathrm{l}$ of resuspended protein A/G plus agarose beads (Santa Cruz Biotechnology, Santa Cruz, CA, USA) at $4{ }^{\circ} \mathrm{C}$ for $1 \mathrm{~h}$. After centrifugation $(1000 \times g, 5 \mathrm{~min})$, the precleared supernatant was collected and the precipitate was used for the detection of MMP-26 as a negative control. Collected supernatant was mixed with $1.5 \mu \mathrm{g}$ of rabbit antiTIMP-4 antibody and $20 \mu \mathrm{l}$ of resuspended protein A/G plus agarose beads at $4^{\circ} \mathrm{C}$ for $4 \mathrm{~h}$. After centrifugation $(1000 \times g, 5 \mathrm{~min})$, the supernatant was collected and this precipitate was also used for the detection of MMP-26. Each precipitate was washed four times with phosphate-buffered saline ( $\mathrm{pH} 7.4$ ) followed by centrifugation (1 000 $\times g, 5 \mathrm{~min}$ ). Separated samples were subjected to further analyses by Western blot and zymography. To examine the interaction between TIMP-4 and MMP-9 or MMP-26 and MMP-9, complexes of rabbit anti-TIMP-4 antibody and protein $\mathrm{A} / \mathrm{G}$ agarose beads or complexes of rabbit anti-MMP-26 antibody and protein $\mathrm{A} / \mathrm{G}$ agarose beads were used to draw down TIMP-4 or MMP-26, respectively.

\section{Gelatin zymography}

Gelatin zymography was performed for MMP-9 detection. For this, $0.5 \mathrm{mg} / \mathrm{ml}$ gelatin was co-polymerized into a $10 \%$ polyacrylamide resolving gel at the time of casting. Complexes of rabbit anti-TIMP-4 antibody and protein $\mathrm{A} / \mathrm{G}$ agarose beads or complexes of rabbit anti-MMP-26 antibody and protein A/G agarose beads or supernatant during immunoprecipitation were then subjected to separation by gelatin-SDS-PAGE. Following electrophoresis, the gel was immersed in buffer containing $0.01 \%$ Brij-35, $10 \mathrm{mM}$ HEPES/NaOH, $10 \mathrm{mM} \mathrm{NaCl}, 10 \mathrm{mM} \mathrm{CaCl}_{2}, 0.1 \mu \mathrm{M} \mathrm{ZnSO}_{4}, \mathrm{pH} 7.5$, at $4{ }^{\circ} \mathrm{C}$ for $12 \mathrm{~h}$ six times to remove SDS and allow enzymatic gelatin substrate hydrolysis. The gel was stained in $0.025 \%$ Coomassie blue in methanol:acetic acid:water (volume ratio of 5:1:4) for $4 \mathrm{~h}$. Finally, the gel was destained with ethanol:acetic acid:water (volume ratio of $1: 1: 8$ ) for 2 days.

\section{Immunohistochemistry}

Formalin-fixed, paraffin-embedded human prostate cancer tissues serially sectioned and fixed on slides were obtained from Mayo Clinic (Jacksonville, FL, USA). All the tissue sections are of the same thickness of $4 \mu \mathrm{m}$. The sections were dewaxed with xylene and rehydrated successively in $100 \%$ ethanol, $95 \%$ ethanol, and distilled water. The slides were then subjected to antigen retrieval by boiling in $0.1 \mathrm{mM}$ citric acid ( $\mathrm{pH} \mathrm{6.0)}$ for $12 \mathrm{~min}$. The samples were then incubated with primary antibody diluted to $25 \mu \mathrm{g} / \mathrm{ml}$ in blocking buffer $(0.2 \%$ Triton X-100, 5\% normal goat serum, and 3\% bovine serum albumin
Table 1 Study population and staining analysis

\begin{tabular}{lcccl}
\hline Pathology & $n$ & $\begin{array}{l}\text { Mean } \\
\text { intensity }\end{array}$ & $\begin{array}{l}\text { Standard } \\
\text { deviation }\end{array}$ & Standard error \\
\hline (a) Statistical & analysis & for MMP-26 reactivity \\
Benign & 32 & 34.92 & 9.85 & 1.74 \\
HGPIN & 27 & 82.01 & 14.81 & 2.85 \\
Cancer & 33 & 61.47 & 12.61 & 2.19 \\
(b) Statistical & analysis & for TIMP-4 reactivity & \\
Benign & 36 & 31.25 & 11.11 & 1.85 \\
HGPIN & 28 & 79.48 & 15.53 & 2.93 \\
Cancer & 38 & 57.72 & 10.39 & 1.69 \\
\hline
\end{tabular}

The mean color intensity and variation in immunohistochemical staining from a population of 41 patient specimens as described in Materials and Methods (see Immunohistochemistry and evaluation and statistical analysis). $n$, sample size (number of patient samples, out of the original 41, in which the designated pathology was observed). Mean intensity represents the mean of the means, while the standard deviation and standard error values are representative of the mean intensity derived from each individual patient sample for any given pathology/category. Please see Results (Study population) for more detailed description.

in TBS) for $1 \mathrm{~h}$ at room temperature. The primary antibodies used were affinity-purified polyclonal rabbit anti-human pro-MMP-26 and TIMP-4 $[12,25]$, or were obtained from commercial sources and derived from different species. Purified preimmune IgG from rabbit was used as a negative control. After the primary antibody was rinsed off with TBS, the tissue sections were incubated with an alkaline phosphatase-conjugated secondary antibody (Sigma) diluted 1:1000 in blocking buffer. Signals were detected by adding Fast Red (Sigma). The sections were counterstained lightly with hematoxylin for viewing of nonreactive cells.

\section{Evaluation and statistical analysis}

Each slide was examined at $400 \times$ using a Nikon Eclipse $50 \mathrm{i}$ microscope with a QImaging 1394 QICAM camera system. With the QcapturePro program v. 5.0, images of the most representative areas of non-neoplastic acini, HGPIN (if present), and cancer were photographed, obtaining three separate JPEG images of each. The images were analyzed on a Windows 2000 PC using the public domain NIH Image program, ImageJ, developed at the National Institutes of Health and available on the Internet (http://rsb.info.nih.gov/nih-image/). For each picture, three spatially separate epithelial cytoplasmic areas, excluding the nuclei, were measured. After an outline had been traced around the area using the freehand spline, the Analyze/Measure function was used to record the mean color intensity within the selected area. The mean intensity of at least three separate areas from each patient sample ( $n$; Table 1 ) was then calculated and normalized to correct for background intensity, and one-way between-subjects analysis of variance was performed for each sample type (Benign, HGPIN, Cancer). Statistical analysis of low-grade prostatic intraepithelial neoplasia (LGPIN) was not feasible because of its insufficient occurrence in the examined tissues. 


\section{Results}

\section{Study population}

The initial study population consisted of 41 patient samples of prostate cancer that had been serially sectioned. The specimens had Gleason scores ranging from 2 to 10 , exclusive of Gleason score 3. As only one patient sample representative of Gleason scores 2 or 10 was available, these samples were excluded from the study and subsequent statistical analyses. Some slides had cancer but not benign tissue, or vice versa. Not every case had HGPIN. Some pathology was not clearly interpretable. In addition, during the immunostaining process some of the tissue sections were dislodged from the slides, and not every sample contained all three types of epithelium, resulting in small fluctuations in the total number of patient samples evaluated in each category. After immunostaining the tissues with antibodies against MMP-26 or TIMP-4, three categories of epithelium were scored based upon staining intensity, showing significant differences $(p<0.0001)$ in paired comparisons of non-neoplastic (benign), HGPIN, and cancer. The characteristics of the specimens evaluated, the resultant mean scores of their normalized values, and their deviations are detailed in Table 1.

\section{Detection of pro- and active forms of MMP-26 by Western blotting}

Unlike other MMPs, pro-MMP-26 proceeds to autoactivation via cleavage sites that are upstream of the cysteine-switch motif $[11,26]$, and $\mathrm{N}$-terminal sequencing of auto-activated MMP-26 reveals several cleavage sites at $\mathrm{Leu}^{49} \downarrow \operatorname{Thr}^{50}[11], \mathrm{Gl}^{59} \downarrow \mathrm{Gl}^{60}[26]$, and $\mathrm{Ala}^{75} \downarrow \operatorname{Leu}^{76}[11]$. Comparison of the antigen peptide sequence designed for the prodomain of MMP-26 (Thr ${ }^{50}$-Gln-Glu-Thr-Gln-ThrGln-Leu-Leu-Gln-Gln-Phe-His-Arg-Asn-Gly-Thr-Asp ${ }^{67}$ ) with the N-terminal sequences of auto-activated MMP-26 reveals that the anti-prodomain MMP-26 antibody can detect both the zymogen and activated forms of MMP-26. Figure 1 demonstrates that both the anti-prodomain and anti-C-terminal MMP-26 antibodies can recognize both the proform and the activated form of bacterially expressed MMP-26. For reasons that are not clear, the anti-C-terminal MMP-26 antibody is more sensitive than the anti-prodomain antibody. Prostate cancer tissue extracts reveal the presence of both pro- and activated MMP-26. The antiprodomain MMP-26 antibody is capable of detecting many forms of activated MMP-26 in the prostate cancer tissue extracts, whereas the anti-C-terminal MMP-26 antibody is able to detect only one form of activated MMP-26. The molecular weights of the activated forms in the tissue extract show little variation from the positive bands for bacterially expressed MMP-26.

\section{Detection of MMP-26 and TIMP-4 complex by co-IP}

Complexes of the rabbit anti-TIMP-4 antibody and protein $\mathrm{A} / \mathrm{G}$ agarose beads were used to pull down TIMP4 from prostate cancer tissue extracts. To examine the possibility of complex formation between MMP-26 and TIMP-4 in these extracts, a goat anti-MMP-26 antibody was used to probe for MMP-26 within the immunoprecipitates and in the retained supernatant. Figure 2 shows positive signals for MMP-26 in the supernatant fraction and perhaps in the anti-TIMP-4 immunoprecipitate. The majority of MMP-26 was found in the supernatant, and a very small fraction of MMP-26 was complexed with TIMP-4, as a very faint MMP-26 band was detected in the anti-TIMP-4 fraction (IP_anti-TIMP-4) when compared to the immunoprecipitates recovered from the preclearing phase (IP_rabbit IgG). A goat polyclonal antibody (E-14, Santa Cruz Biotechnology) against near the C-terminal sequence region of MMP-26 reveals a band similar to that observed with the rabbit anti-C-terminal MMP-26 antibody. Although antibodies from different species were utilized, a mouse alkaline phosphatase-conjugated anti-goat secondary antibody interacted with the rabbit antibody, resulting in the detection of the heavy (above $48 \mathrm{kDa}$ ) and light (27 $\mathrm{kDa}$ ) chains of rabbit IgG.

\section{Detection of pro- and active MMP-9 by gelatin zymography} Expression of MMP-9 from prostate cancer tissue ex-

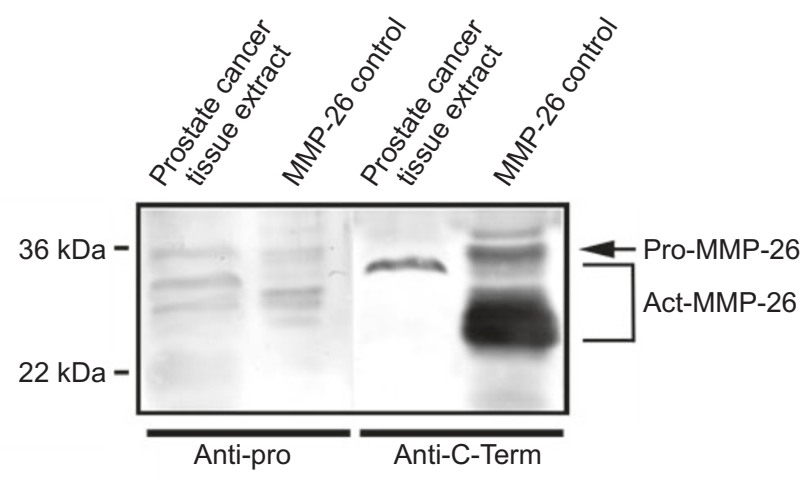

Figure 1 Western blot MMP-26 detection from human prostate cancer tissue extracts using rabbit anti-prodomain and anti-C-terminal MMP-26 antibodies. Bacterially expressed MMP-26 was used as a control. Sequence comparisons between the MMP-26 prodomain epitope and N-terminal analyses of activated MMP-26 shows overlap of those peptide sequences. As a result, the anti-pro-MMP-26 antibody recognizes both the pro- and activated (act-) forms of MMP-26. The anti-C-terminal MMP-26 antibody demonstrates a higher sensitivity for MMP-26 than the anti-pro-MMP-26 antibody, but recognizes only a single active form in the tissue extract. 
tracts was not detected by Western blot analysis perhaps due to its low concentration or low antibody sensitivity (data not shown). Precipitated TIMP-4/antibody/protein A/G complexes or MMP-26/antibody/protein A/G complexes were used for zymography. MMP-9 was not detected in either the TIMP-4 precipitate (IP_anti-TIMP-4) or the MMP-26 precipitate (IP_anti-MMP-26). Pro- and active MMP-9 remained in the supernatants (TIMP-4 supernatant, MMP-26 supernatant) (Figure 3). Expression levels of MMP-9 were very low in these prostate cancer tissues. The majority of MMP-9 presented as the proform with very little activated MMP-9 detected.

\section{Immunohistochemistry to detect MMP-26, TIMP-4, and MMP-9}

Immunohistochemical analysis revealed that MMP-26 was present in the prostatic epithelium as well as in the surrounding stroma (Figure $4 \mathrm{~A}-\mathrm{C}$ ). The data showed that MMP-26 expression in HGPIN and cancer was significantly higher than that seen in non-neoplastic epithelia $(p<0.0001)$. Furthermore, the expression of MMP-26 was maximal in HGPIN, and was found to be expressed at significantly lower levels $(p<0.001)$ in cancer within the same tissue specimens (Figure 5A). MMP-26 expression in cancer displayed no correlation with Gleason score during this study (data not shown). The expression of TIMP-4 was also detected in the prostatic epithelium as well as in

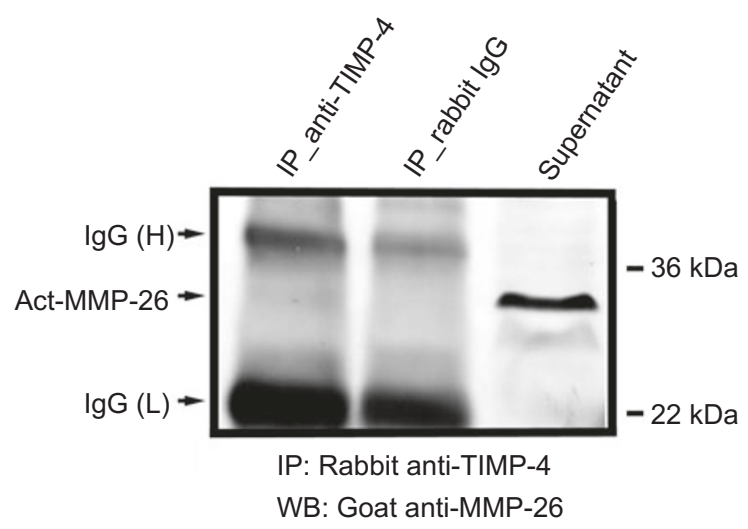

Figure 2 Western blot (WB) of immunoprecipitates (IP). TIMP-4 was drawn down using rabbit anti-TIMP-4 antibodies, and the precipitates were then probed with a goat anti-MMP-26 antibody against the C-terminal. A strong MMP-26 band was detected in the retained supernatant fraction (supernatant), while a very weak band was detected in the precipitate (IP_anti-TIMP-4). Blotting of the precipitates from preclearing with rabbit preimmune $\operatorname{IgG}$ were completely clear (IP_rabbit IgG). In both cases, light $(27 \mathrm{kDa})$ and heavy $(48 \mathrm{kDa})$ $\mathrm{IgG}$ chains, i.e. $\operatorname{IgG}(\mathrm{L})$ and $\operatorname{IgG}(\mathrm{H})$, were also visualized.

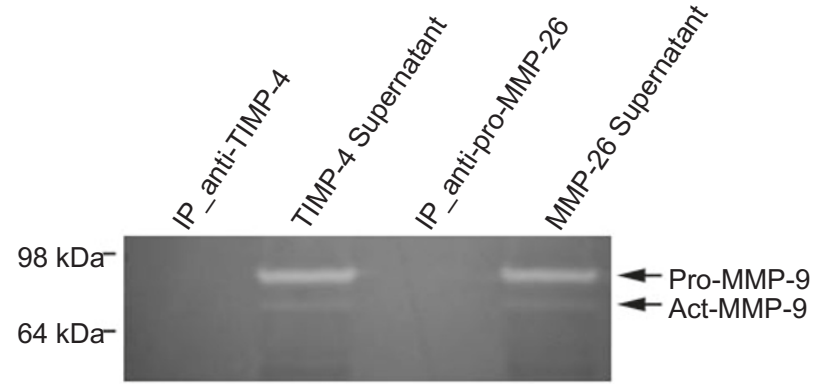

Figure 3 Gelatin zymography of TIMP-4 immunoprecipitate from prostate cancer tissue extracts to detect MMP-9. Gelatin $(0.5$ $\mathrm{mg} / \mathrm{mL}$ ) was co-polymerized into a $10 \%$ polyacrylamide resolving gel. IP_anti-TIMP-4 and IP_anti-pro-MMP-26 designate the precipitates following immunoprecipitation with anti-TIMP-4 and anti-pro-MMP-26 antibodies, respectively, in the presence of protein A/G agarose beads. TIMP-4 supernatant and MMP-26 supernatant designate the retained supernatant following the procedures detailed above. MMP-9 was detected only in the supernatant, and failed to form a complex with either the TIMP-4 or MMP-26 antibodies.

the surrounding stroma (Figure 4D-F), in a pattern that mimicked that of MMP-26. TIMP-4 expression in HGPIN and cancer was again significantly higher than that seen in non-neoplastic epithelium $(p<0.0001)$, and furthermore, the expression of TIMP-4 was highest in HGPIN, with significantly reduced expression $(p<0.0001)$ in cancer within the same tissue specimen (Figure 5B). As before, the expression of TIMP-4 in HGPIN and cancer was not correlated with Gleason score (data not shown). Prostate cancer tissue revealed no positive signals for the expression of MMP-9 using a mouse monoclonal antibody against MMP-9 (Ab-1) from Calbiochem (data not shown), in accordance with the low levels of MMP-9 expression seen during the gelatin zymography assays.

\section{Semi-colocalization between TIMP-4 and MMP-26}

Serial sections of prostate cancer tissue were used to determine if any areas of colocalization exist between MMP-26 and TIMP-4. Figure 6 shows areas of high TIMP4 expression that correspond to areas of high MMP-26 expression, suggesting colocalization between these enzymes, while adjacent control sections stained with rabbit preimmune $\mathrm{IgG}$ produced no positive signals.

\section{Discussion}

MMPs and TIMPs have been associated with invasion and metastasis in many human cancers, and evidence is growing that MMP-26 and TIMP-4 are important factors 
A

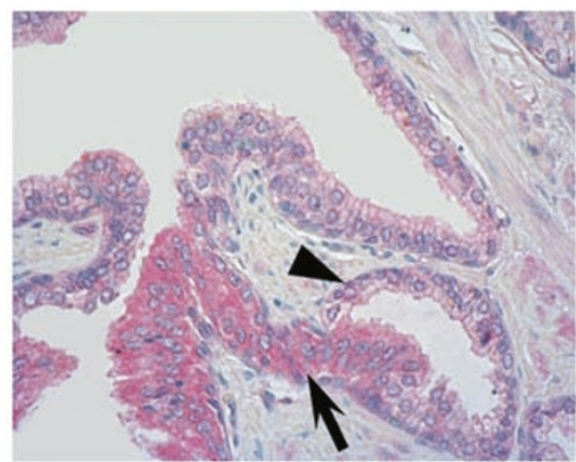

C

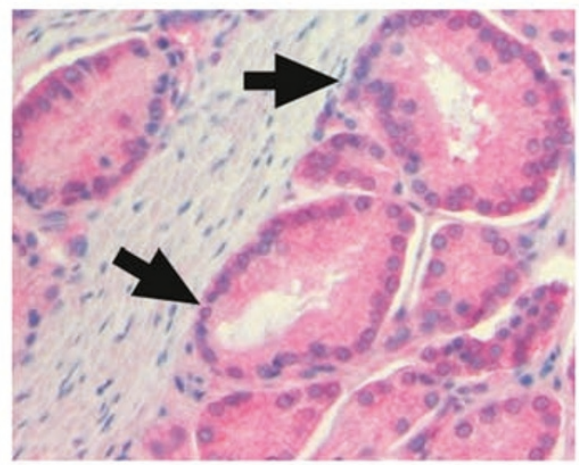

$\mathrm{E}$

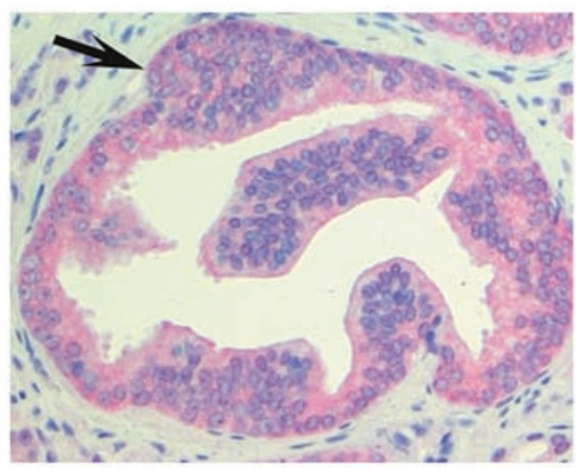

B

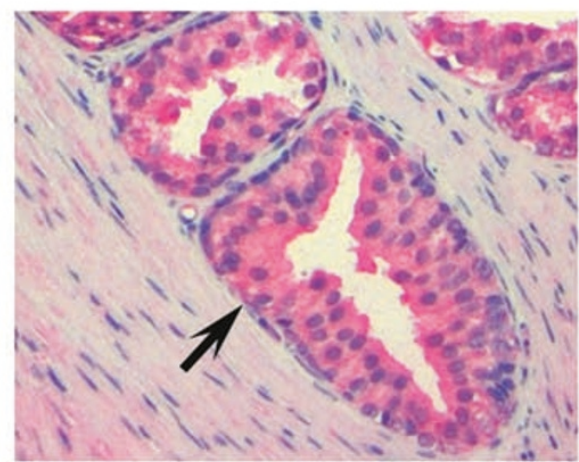

D

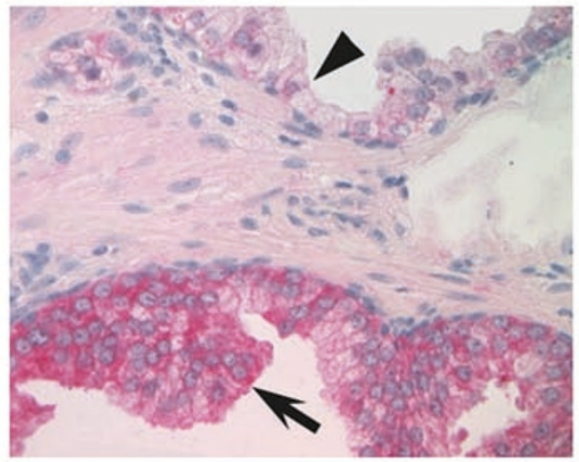

$\mathrm{F}$

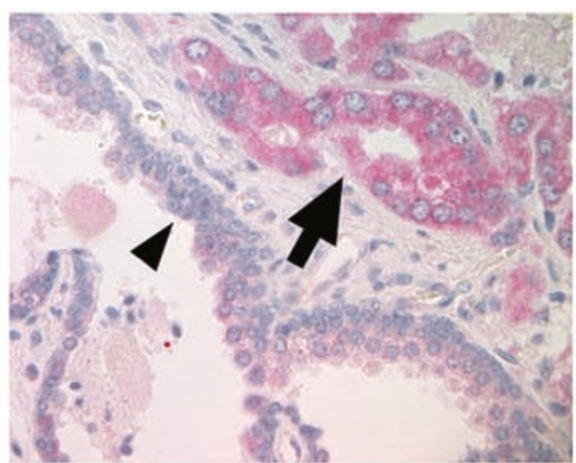

Figure 4 MMP-26 and TIMP-4 immunohistochemical staining in non-neoplastic epithelium (arrowhead), HGPIN (thin arrow), and cancer (thick arrow). (A-C) MMP-26 immunostaining. (A) HGPIN is stained more intensely than the adjacent non-neoplastic epithelium. The areas left and above the region of HGPIN are non-neoplastic tissues representing partial glandular involvement of a benign acinus; (B) another example of intense HGPIN staining; and (C) positive staining in cancer tissues. (D-F) TIMP-4 immunostaining. (D) HGPIN is stained more intensely than non-neoplastic epithelium; (E) a second example of intense HGPIN staining; and (F) regions of cancer exhibit more intense staining than non-neoplastic epithelium. All pathologic diagnoses were performed by $\mathrm{KAI}$ in accordance with established criteria [27].

during prostate cancer progression. The epithelium of normal and preinvasive human prostate tissues is physically separated from the stroma by two continuous layers: first by basal cells, and second, by a basement membrane. Disruption of both the basal cell layer and the basement membrane are prerequisites for carcinoma cell invasion. In this study, we show that the levels of MMP-26 and TIMP-4 in human prostate tissues are significantly higher in HGPIN than either non-neoplastic epithelium or cancer. In addition, we show that the levels of MMP-26 and TIMP-4 reach their 


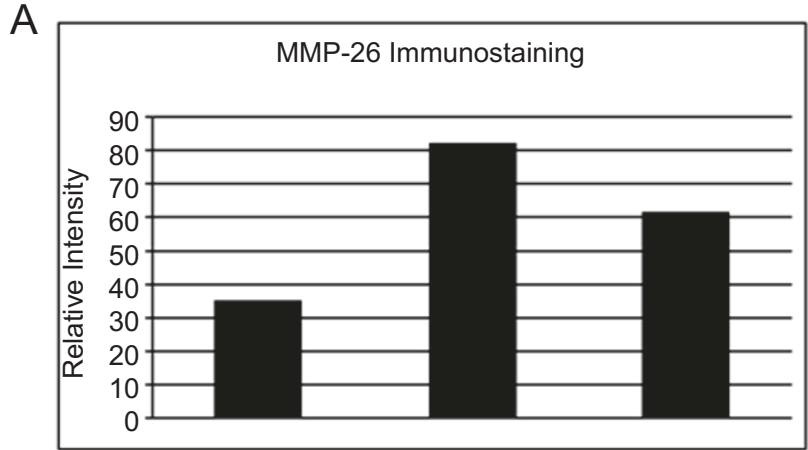

B

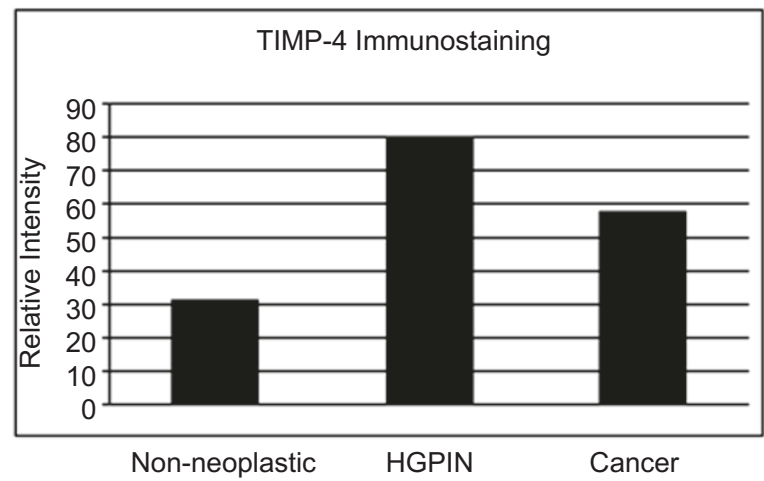

Figure 5 Analyses of MMP-26 and TIMP-4 immunostaining in non-neoplastic ducts and acini, HGPIN, and cancer. (A) MMP-26 immunostaining. HGPIN stained more intensely than non-neoplastic epithelium $(p<0.0001)$ or cancer $(p<0.001)$ in the same tissues, with the least intense staining observed in benign acini. (B) TIMP-4 immunostaining. Findings mimicked those of MMP-26, with HGPIN staining more intensely than non-neoplastic epithelium $(p<0.0001)$ or cancer $(p<0.0001)$, and the least intense staining again found in benign acini. Notably, significant differences $(p<0.0001)$ were found between every pair of tissue categories analyzed. Standard error values were of such small magnitude as to warrant their omission (see Table 1). maximum in HGPIN, then decline significantly as a duct or acinus progresses from HGPIN to cancer. Furthermore, the parallel expression patterns of MMP-26 and TIMP-4 indicate that they are highly coordinated throughout malignant transformation.

MMP-26 is a multifaceted enzyme with many direct ECM targets as well as non-ECM protein targets including insulin-like growth factor-binding protein-1 and $\alpha-1$ protease inhibitor [7-10]. Previous results from our group revealed that MMP-26 promoted the invasion of human prostate cancer cells through both type IV collagen and fibronectin, not only through the direct proteolytic cleavage of these proteins but also through the activation of pro-MMP-9, a highly efficient enzyme with multiple targets in the ECM [12]. While these current results show that the expression of MMP-9 in prostate cancer tissues was not detectable by Western blot, low levels of MMP-9 expression were detected by zymography, primarily in the zymogen form.

TIMP-4 is a potent inhibitor of MMP-26 [7, 13], and transfection of human breast cancer cells with TIMP-4 cDNA showed that TIMP-4 could significantly inhibit cancer cell invasion across a Matrigel barrier [20]. Furthermore, our group has shown that MMP-26 and TIMP-4 are colocalized in human breast carcinoma tissue [13]. While our current co-IP studies show only scant evidence of TIMP-4/MMP-26 complex formation, immunostaining of serially sectioned prostate cancer tissues does reveal areas of possible colocalization between MMP-26 and TIMP-4. TIMP-4 is a tight-binding and slow-binding inhibitor of MMP-26 in vitro, with an apparent $K_{\mathrm{i}}$ value of $0.62 \mathrm{nM}$ [13], but in vivo, and during the co-IP analyses, the presence of additional proteins might interfere with the formation of a TIMP-4/MMP-26 complex. Taken as a whole, these findings combine to suggest that MMP-26 and TIMP-4 might

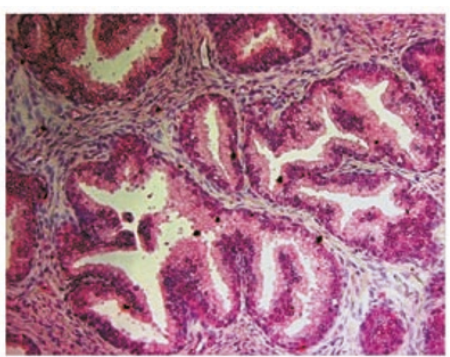

TIMP-4

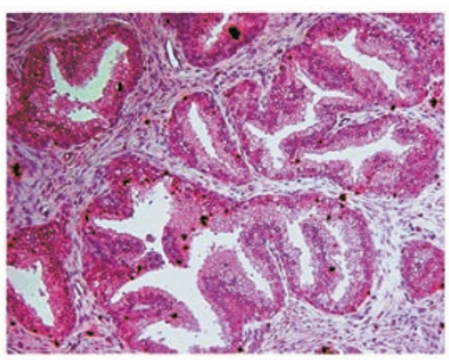

MMP-26

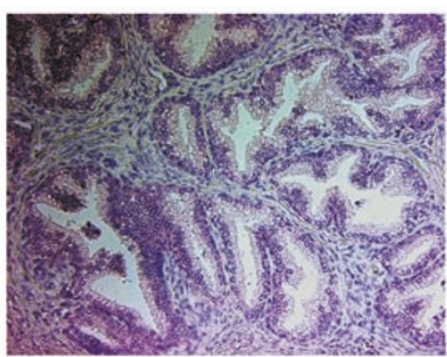

Control

Figure 6 Immunohistochemical staining of serially sectioned human prostate cancer tissues containing HGPIN reveals similar patterns of staining for TIMP-4 and MMP-26 on adjacent sections. Rabbit preimmune IgG control using another adjacent section was negative. 
participate in interactions that ultimately affect both the surrounding ECM and the activity of other proteins critical to the progression of in vivo tumor growth.

The finding that the levels of MMP-26 and TIMP-4 are highest in HGPIN is significant because HGPIN is considered the preinvasive precursor of prostate cancer. HGPIN shares malignant cytologic features with cancer, but lacks the stromal invasion that characterizes cancer. The progression of HGPIN to cancer is associated with progressive disruption of the basal cell layer and focal loss of the basement membrane [27]. Our current findings are analogous to our previous studies in breast cancer that show the highest expression of MMP-26 and TIMP-4 in DCIS, with lower expression present in normal breast epithelium and invasive cancer [13]. Prostatic HGPIN is the preinvasive analogue of DCIS. These data also correlate with studies of endometrial cancer, where MMP-26 and TIMP-4 expression was shown to decrease with the loss of histologic differentiation $[18,28]$. Furthermore, our study can be correlated with an investigation of squamous cell cancer (SCC), which found the highest expression of MMP-26 in low-grade SCC and an absence of MMP-26 expression in dedifferentiated high-grade SCC [29]. Taken together, these data indicate that MMP-26 and TIMP-4 may play important roles in facilitating the initial conversion to an invasive phenotype.

Disruption of the basement membrane is one of the final steps that allow pathologically transformed epithelial cells to become invasive. The basement membrane or basal lamina that encircles a normal or preinvasive human prostate duct or acinus is composed of proteins that include laminin, type IV collagen, entactin, glycosaminoglycans, and heparin sulfate proteoglycans. MMP-26, in conjunction with one of its cleavage products, active MMP-9, efficiently cleaves many components of the basement membrane [12]. MMP-26 may be able to gain access to cleavage sites within the basement membrane through focal disruptions in the basal cell layer. The maximal expression of MMP26 in HGPIN, with subsequent reduced levels in cancer, indicates that this enzyme may be temporally regulated. As MMP-26 expression is lost, invasive tumors may then begin expressing other enzymes better suited for late-stage invasion and metastasis.

The observed trend in TIMP-4 expression mimicked that of MMP-26, with its highest expression in HGPIN and significantly decreased expression in cancer. This parallel protein regulation differs from many previous studies that showed differential expression patterns between MMPs and TIMPs in human prostate cancer, and may indicate the existence of different regulatory mechanisms among the TIMPs. One hypothesis for the coordinated expression of MMP-26 and TIMP-4 is that as the expression of the weaker inhibitors of MMP-26 decline, TIMP-4 may be left as the sole compensatory mechanism to overcome the increased activity of MMP-26. The increase in the levels of MMP-26 may correlate with the increased expression of TIMP-4 in a positive feedback manner so that the pathologically upregulated activity of MMP-26 might require a higher set-point of TIMP-4. We have recently reported a similar coordinated expression pattern of MMP-26 and TIMP-4 in human breast cancer [13], and co-regulation between MMP-26 and TIMP-4 in cycling endometrium and in endometrial cancer has also been reported $[18,28$, 30]. Progression to invasive cancer may occur when the upregulation of TIMP-4 in response to increased levels MMP-26 is no longer able to counteract the proinvasive influence of MMP-26. Once the basement membrane has been ruptured, however, cancer cells may become less dependent upon the activity of MMP-26, resulting in a decline in the expression of this enzyme along with that of its highly coordinated inhibitor, TIMP-4.

The present study indicates that MMP-26 and TIMP4 may play important roles in the initial conversion of prostate cancer cells to an invasive phenotype, and that these two proteins are highly coordinated throughout this transformation. The high levels of MMP-26 and TIMP-4 in HGPIN suggest their use as biomarkers for the early diagnosis of a subset of patients with HGPIN before there is invasive tumor and metastasis. The timely identification of early-stage prostate tumor might lead to novel strategies for treatment that would halt or delay the progression of the disease. Although the implications of the coordinately high expression of MMP-26 and TIMP-4 in human HGPIN are not completely understood, this study reveals the importance and necessity of future studies into the complex roles and interactions of MMP-26 and TIMP-4 in human prostate cancer.

\section{Acknowledgments}

This work was supported in part by grants from DOD/ US Army Prostate Cancer Research Program DAMD1702-1-238, Elsa U Pardee Foundation, an FSU Developing Scholar Award (to Q-XAS), and Program Enhancement Grants from Florida State University Research Foundation (to Q-XAS and Y-G Z).

\section{References}

1 Nelson AR, Fingleton B, Rothenberg ML, Matrisian LM. Matrix metalloproteinases: biologic activity and clinical implications. J Clin Oncol 2000; 18:1135-1149.

2 Sternlicht MD, Werb Z. How matrix metalloproteinases regulate cell behavior. Annu Rev Cell Dev Biol 2001; 17:463-516.

3 Egeblad M, Werb Z. New functions for the matrix metalloprotein- 
ases in cancer progression. Nat Rev Cancer 2002; 2:163-176.

4 Kugler A. Matrix metalloproteinases and their inhibitors. Anticancer Res 1999; 19:1589-1592.

5 Stetler-Stevenson WG. Matrix metalloproteinases in angiogenesis: a moving target for therapeutic intervention. J Clin Invest 1999; 103:1237-1241.

6 Chambers AF, Matrisian LM. Changing views of the role of matrix metalloproteinases in metastasis. J Natl Cancer Inst 1997; 89:1260-1270.

7 Uria JA, Lopez-Otin C. Matrilysin-2, a new matrix metalloproteinase expressed in human tumors and showing the minimal domain organization required for secretion, latency, and activity. Cancer Res 2000; 60:4745-4751.

8 Park HI, Ni J, Gerkema FE, et al. Identification and characterization of human endometase (matrix metalloproteinase-26) from endometrial tumor. J Biol Chem 2000; 275:20540-20544.

9 de Coignac AB, Elson G, Delneste Y, et al. Cloning of MMP26. A novel matrilysin-like proteinase. Eur J Biochem 2000; 267:3323-3329.

10 Marchenko GN, Ratnikov BI, Rozanov DV, et al. Characterization of matrix metalloproteinase-26, a novel metalloproteinase widely expressed in cancer cells of epithelial origin. Biochem $\mathrm{J}$ 2001; 356:705-718.

11 Park HI, Turk BE, Gerkema FE, et al. Peptide substrate specificities and protein cleavage sites of human endometase/matrilysin2/matrix metalloproteinase-26. J Biol Chem 2002; 277:3516835175.

12 Zhao YG, Xiao AZ, Newcomer RG, et al. Activation of progelatinase $\mathrm{B}$ by endometase/matrilysin- 2 promotes invasion of human prostate cancer cells. J Biol Chem 2003; 278:15056-15064.

13 Zhao YG, Xiao AZ, Park HI, et al. Endometase/matrilysin-2 in human breast ductal carcinoma in situ and its inhibition by tissue inhibitors of metalloproteinases- 2 and -4 : a putative role in the initiation of breast cancer invasion. Cancer Res 2004; 64:590598.

14 Carmichael DF, Sommer A, Thompson RC, et al. Primary structure and cDNA cloning of human fibroblast collagenase inhibitor. Proc Natl Acad Sci USA 1986; 83:2407-2411.

15 Stetler-Stevenson WG, Brown PD, Onisto M, et al. Tissue inhibitor of metalloproteinases-2 (TIMP-2) mRNA expression in tumor cell lines and human tissues. J Biol Chem 1990; 265:13933-13938.

16 Uria JA, Ferrando AA, Velasco G, et al. Structure and expression in breast tumors of human TIMP-3, a new member of the metalloproteinase inhibitor family. Cancer Res 1994; 54:2091-2094.

17 Greene J, Wang M, Liu YE, et al. Molecular cloning and characterization of human tissue inhibitor of metalloproteinase 4. J Biol Chem 1996; 271:30375-30380.
18 Pilka R, Domanski H, Hansson S, et al. Endometrial TIMP-4 mRNA is high at midcycle and in hyperplasia, but down-regulated in malignant tumours. Coordinated expression with MMP26. Mol Hum Reprod 2004; 10:641-650.

19 Zhang J, Cao YJ, Zhao YG, et al. Metalloproteinase-26 (MMP26) and tissue inhibitor of metalloproteinase 4 (TIMP-4) in human normal cytotrophoblast cells and a choriocarcinoma cell line, JEG-3. Mol Hum Reprod 2002; 8:659-666.

20 Wang W, Liu YE, Greene J, et al. Inhibition of tumor growth and metastasis of human breast cancer cells transfected with tissue inhibitor of metalloproteinase 4. Oncogene 1997; 14:2767-2774.

21 Liu YE, Wang M, Greene J, et al. Preparation and characterization of recombinant tissue inhibitor of metalloproteinase 4 (TIMP-4). J Biol Chem 1997; 272:20479-20483.

22 Liu AY, Zhang H, Sorensen CM, Diamond DL. Analysis of prostate cancer by proteomics using tissue specimens. J Urol 2005; 173:73-78.

23 Lichtinghagen R, Musholt PB, Stephan C, et al. mRNA expression profile of matrix metalloproteinases and their tissue inhibitors in malignant and non-malignant prostatic tissue. Anticancer Res 2003; 23:2617-2624.

24 Brehmer B, Biesterfeld F, Jakse G. Expression of matrix metalloproteinases (MMP-2 and -9) and their inhibitors (TIMP-1 and -2) in prostate cancer tissue. Prostate Cancer Prostat Dis 2003; 6:217-222.

25 Hurst DR, Li H, Xu X, et al. Development and characterization of a new polyclonal antibody specifically against tissue inhibitor of metalloproteinases 4 in human breast cancer. Biochem Biophys Res Commun 2001; 281:166-171.

26 Marchenko ND, Marchenko GN, Strongin AY. Unconventional activation mechanisms of MMP-26, a human matrix metalloproteinase with a unique PHCGXXD cysteine-switch motif. J Biol Chem 2002; 277:18967-18972.

27 Bostwick DG, Qian J. High-grade prostatic intraepithelial neoplasia. Mod Pathol 2004; 17:360-379.

28 Pilka R, Norata GD, Domanski H, et al. Matrix metalloproteinase26 (matrilysin-2) expression is high in endometrial hyperplasia and decreases with loss of histological differentiation in endometrial cancer. Gynecol Oncol 2004; 94:661-670.

29 Ahokas K, Skoog T, Suomela S, et al. Matrilysin-2 (matrix metalloproteinase-26) is upregulated in keratinocytes during wound repair and early skin carcinogenesis. J Invest Dermatol 2005; 124:849-856.

30 Tunuguntla R, Ripley D, Sang QX, Chegini N. Expression of matrix metalloproteinase-26 and tissue inhibitors of metalloproteinases TIMP-3 and -4 in benign endometrium and endometrial cancer. Gynecol Oncol 2003; 89:453-459. 